\title{
Protocol of the randomized control trial: the WiseApp trial for improving health outcomes in PLWH (WiseApp)
}

\author{
Gabriella Flynn ${ }^{1}$, Haomiao Jia ${ }^{2}$, Nancy R. Reynolds ${ }^{3}$, David C. Mohr ${ }^{3,4}$ and Rebecca Schnall ${ }^{2 *}$ (D
}

\begin{abstract}
Background: Poor adherence to antiretroviral therapy (ART) is one of the primary barriers to viral load suppression. mHealth technology can help overcome challenges with ART adherence. This paper outlines the protocol for the WiseApp randomized control trial. The WiseApp contains real-time medication monitoring linking an electronic pill bottle and fitness tracker to the app, helping persons living with HIV (PLWH) self-manage their medication adherence and improve their overall quality of life. The primary objective of the trial is to test the effect of the WiseApp's medication adherence features on antiretroviral adherence in underserved PLWH in New York City.

Methods: This ongoing study is a two-arm randomized control trial. Participants are randomized 1:1 to the WiseApp intervention arm or the control arm at baseline and followed for 6 months. Eligibility criteria include: 18 years of age, have a diagnosis of HIV, speak and understand English or Spanish, live in the United States, own a smartphone, currently taking ART medications, and report the past 30 days adherence of $80 \%$ or less as measured using the Visual Analogue Scale (VAS), or have a viral load of over 400 copies $/ \mathrm{mL}$. The sample size for the trial is 200 people. All study participants receive the WiseApp, a CleverCap electronic pill bottle, and a fitness tracker. The intervention group also receives videos and health surveys centered on medication adherence and managing living with HIV as well as medication reminders. In contrast, the control group receives walk step reminders, videos, and surveys focused on overall wellness.

Discussion: The WiseApp Trial has the potential to improve HIV self-management applications, being one of the few randomized controlled trials of a mHealth medication adherence and HIV self-management application in the United States. The trial could also bring new opportunities for advancement in reaching economically disenfranchised and underserved populations in the United States. The real-time monitoring of the WiseApp has the potential to help providers initiate interventions to help patients resume treatment before drug resistance begins.
\end{abstract}

Trial registration: This trial was registered with ClinicalTrials.gov (NCT03205982) on July 2, 2017.

Keywords: HIV/AIDS, Mobile health, Medication adherence, Randomized controlled trial, Real-time monitoring

\footnotetext{
* Correspondence: rb897@columbia.edu

${ }^{2}$ Division of Scholarship and Research, Columbia University School of

Nursing, 560 West 168th Street, New York, NY 10032, USA

Full list of author information is available at the end of the article
}

(c) The Author(s). 2020 Open Access This article is licensed under a Creative Commons Attribution 4.0 International License, which permits use, sharing, adaptation, distribution and reproduction in any medium or format, as long as you give appropriate credit to the original author(s) and the source, provide a link to the Creative Commons licence, and indicate if changes were made. The images or other third party material in this article are included in the article's Creative Commons licence, unless indicated otherwise in a credit line to the material. If material is not included in the article's Creative Commons licence and your intended use is not permitted by statutory regulation or exceeds the permitted use, you will need to obtain permission directly from the copyright holder. To view a copy of this licence, visit http://creativecommons.org/licenses/by/4.0/ The Creative Commons Public Domain Dedication waiver (http://creativecommons.org/publicdomain/zero/1.0/) applies to the data made available in this article, unless otherwise stated in a credit line to the data. 


\section{Background}

An estimated 1.1 million people in the U.S. are living with HIV, with the epidemic concentrated in racial and ethnic minorities [1]. Over time, with the development of effective antiretroviral treatments, HIV has progressed to being a chronic condition with people's life expectancy now being measured in decades [2]. However, in order for HIV to be successfully managed and maintained, persons living with HIV (PLWH) need to have access to care, begin treatment, remain in care and adhere to their antiretroviral therapy (ART) [2].

In the U.S., it is estimated that for every $100 \mathrm{PLWH}$, only 28 people successfully complete the steps to manage their care and stay in treatment with poor adherence to ART remaining the most significant challenge to treatment success $[3,4]$. Insufficient engagement in care and adherence to HIV treatment leads to the progression of HIV disease and premature death among PLWH $[5,6]$. New York City (NYC) accounts for $82 \%$ of all PLWH in New York State, and in the NYC region only $67 \%$ of PLWH achieve viral suppression from successful adherence to antiretroviral therapy [7]. Despite disparate populations being the most likely to be infected with HIV, these populations are less likely to adhere to their medication regimens [8]. Creating effective interventions to prevent loss of virologic control, drug resistance, and loss of treatment options as a result of non-adherence is crucial $[9,10]$.

mHealth technology can be useful for the management of chronic illnesses, including HIV, helping overcome the challenges of adherence to ART [11]. Further, given the ubiquity of mobile devices, mHealth has the potential to be an effective platform for the delivery of medication adherence interventions [12]. In response, we designed the WiseApp, a self-management application (app) that contains real-time medication monitoring, linking an electronic pill bottle and fitness tracker to the app, helping PLWH self-manage their medication adherence and improve their overall quality of life. The WiseApp draws on formative work with HIV Clinicians, PLWH, HIV Case Managers and the Centers for Disease Control and Prevention [13]. We refined the app for PLWH through a three-step usability evaluation using a think-aloud protocol with end-users, heuristic evaluations, and an end-user cognitive walkthrough [14]. The WiseApp is currently being tested in a randomized control trial (RCT) with PLWH in New York City and registered with ClinicalTrials.gov (NCT03205982).

\section{Study objective}

The primary objective of this RCT was to test the effect of the WiseApp's medication adherence features on ART adherence in underserved PLWH in NYC [14].
This paper provides an overview of the WiseApp RCT protocol.

\section{Ethics and consent}

The Columbia University Institutional Review Board reviewed and approved all study procedures. Participants provided written consent and HIPAA authorization before enrollment, and all data collection and management procedures are collected in accordance with University policy.

\section{Methods/design Study design}

This study is a two-arm RCT among racially and ethnically diverse underserved PLWH. Participants are randomized to the WiseApp intervention arm or the control arm at baseline and followed for 6 months. The differences between intervention and control arms are summarized in the description of the intervention section and outlined in Table 1.

\section{Recruitment and eligibility}

Recruitment consists primarily of community outreach, posting flyers as well as word of mouth. Community outreach is conducted in the five boroughs of NYC, visiting various community and HIV resource centers, and distributing flyers in underserved communities. Flyers also distributed at New York-Presbyterian, and the WiseApp project is listed on the Columbia University Irving Medical Center's (CUIMC) RecruitME website, a CUIMC research database listing all actively enrolling research studies at CUIMC. Study participants also receive a study flyer to share with individuals in their community.

In order to be eligible, participants must be 18 years of age and older, have a diagnosis of HIV, speak and understand English or Spanish, live in the U.S., own a smartphone, and currently take ART medications. Additionally, participants must report the past 30 days adherence of $80 \%$ or less as measured using the Visual Analogue Scale (VAS) or have a viral load of over 400 copies/mL. Exclusion criteria include participation in any other mobile app study for PLWH, currently participating in a directly observed therapy program, not passing the mini-mental state neurocognitive assessment, and an inability to use apps on

Table 1 Comparison of Intervention vs. Control Group

\begin{tabular}{lll}
\hline & Intervention & Control \\
\hline The Wise App & & \\
• Medication Reminders & $X$ & \\
The WiseApp & & $X$ \\
- Step Goal Reminder & $X$ & $X$ \\
\hline CleverCap & \\
\hline
\end{tabular}


their phone [15]. Before participating in any study screening procedures, participants must voluntarily provide verbal informed consent. If found eligible after prescreening, participants provide written consent for the RCT during their first study visit before beginning study procedures. Our goal is to enroll 200 PLWH with 172 participants currently enrolled in the trial. We project that we will close recruitment in the Summer of 2021. (Fig. 1).

\section{Randomization}

Study participants are randomized (1:1) to the WiseApp intervention or the control arm with a variable permuted randomized block design with the block size randomly selected between blocks of four to eight [16]. The treatment assignments in the block design were predetermined before beginning the RCT and have remained static throughout the trial. Random assignments are concealed from the participants for the duration of their participation while study staff members are aware of the participant's randomization at baseline. At the baseline visit, study staff open a sealed study envelope and create the WiseApp profile that aligns with the treatment assignment listed inside the envelope for the baseline visit.

\section{Description of intervention: the WiseApp}

All study participants receive the WiseApp, a CleverCap electronic pill bottle, and a fitness tracker (Fig. 2). The WiseApp originated from formative work to design a self-management app for PLWH and was guided by Fogg's functional triad for computing technology model $[17,18]$. The WiseApp includes the following functional components: testimonials of lived experiences, push-notification reminders, medication trackers, health surveys, chat rooms, and a "To-Do" list outlining tasks for the day. Both study arms receive a fitness tracker that connects to the WiseApp. In the WiseApp, both arms also have a history tab to monitor whether or not they completed their assigned daily goals. Both study arms also receive the CleverCapp pill bottle, with only the intervention group linking the pill bottle to the WiseApp.

The control group receives videos and health surveys centered on wellness and promoting a healthy lifestyle through diet, sleep, exercise, etc. Their "To Do" list consists of daily walk step and completing their weekly videos and surveys. Researchers can track a control member's usage of the pill bottle through the CleverCap platform. Their history tab shows the control group's adherence to meeting their daily step goal of 5000 steps per day. The goal of 5000 steps was selected as less than 5000 steps is often used as a sedentary lifestyle index that can be associated with cardiometabolic risks [19,
20]. The control group receives daily reminders to meet the walk step goals.

\section{Study assessments}

Participant complete a survey at each study visit administered through Qualtrics software. All study data are securely stored in a limited access database by study ID. All hard copy participant information (e.g., study checklists, consent forms) are securely stored at each study site in locked file cabinets with limited access. Participants are enrolled in person and complete three inperson study assessment visits over their 6 months in the RCT. At Baseline, 3 month, and 6 month follow-ups, participants have their blood drawn to measure CD4 and viral load. Participants also have the option of providing recent lab results of their $\mathrm{CD} 4$ and viral load data taken within the last 1-2 weeks.

In addition to completing a blood draw, participant height, weight, hip, and waist measurements are collected following standard NHANES waist and hip measurement protocol [31, 32]. Furthermore, participants complete a survey on an iPad. A full list of the study measures and tools with their measurement time points are listed in Table 2.

\section{Outcomes}

The primary outcome of the RCT is ART adherence. The CleverCap pill dispenser is the primary tool used to measure medication adherence. In addition, we use the Center for Adherence Support Evaluation (CASE) Adherence Index, and CD4 and viral load values to estimate ART adherence. The CleverCap pill dispenser automatically records each time a participant opens the pill bottle. Data collection on the opening of the pill bottle is captured each day for 6 months. To validate the findings from the CleverCap dispenser, both CD4 and $\mathrm{VL}$ are processed from the blood draw at each visit, and participants complete a self-report measure for adherence using the CASE Adherence Index. This adherence index consists of three questions with items scoring at a higher value indicating better adherence [44]. The secondary outcomes are: healthcare access, system use, technology acceptance, and physical activity measures. A list of all the tools to measure the secondary outcomes are listed above in Table 2.

\section{Statistical analysis \\ Sample size calculation}

The targeted enrollment is 200 PLWH who are less than $80 \%$ adherent to their ART medications. We estimate that this will have greater than $80 \%$ power to detect at least a $10 \%$ difference in adherence to ART medication between the WiseApp intervention and the control arm. We made the following assumptions in our sample size 


\begin{tabular}{|c|c|c|c|c|}
\hline & \multicolumn{4}{|c|}{ STUDY PERIOD } \\
\hline & Enrolment & Allocation & Post-allocation & $\begin{array}{c}\text { Close-out } \\
\text { (Apps Removed) }\end{array}$ \\
\hline TIMEPOINT & $-t_{1}$ & 0 & $3 m o$ & $6 \mathrm{mo}$ \\
\hline \multicolumn{5}{|l|}{ ENROLMENT: } \\
\hline Informed consent & $\mathrm{X}$ & & & \\
\hline Allocation & & $\mathrm{X}$ & & \\
\hline \multicolumn{5}{|l|}{ INTERVENTIONS: } \\
\hline $\begin{array}{l}\text { Both groups receive CleverCap } \\
\text { pill dispenser \& fitness tracker }\end{array}$ & & $\mathrm{X}$ & & \\
\hline App download for both groups & & $X$ & & \\
\hline \multicolumn{5}{|l|}{$\begin{array}{r}\text { Medication reminders for } \\
\text { intervention group only }\end{array}$} \\
\hline \multicolumn{5}{|l|}{$\begin{array}{r}\text { Physical Activity Reminders for } \\
\text { control group only }\end{array}$} \\
\hline \multicolumn{5}{|l|}{ ASSESSMENTS: } \\
\hline \multicolumn{5}{|l|}{$\begin{array}{r}\text { Socio-demographic } \\
\text { characteristics, health literacy, } \\
\text { social desirability }\end{array}$} \\
\hline $\begin{array}{r}\text { Behavioral data, Health status, } \\
\text { Physical health measures, } \\
\text { housing status, ART adherence, } \\
\text { Health care access, technology } \\
\text { acceptance }\end{array}$ & & $\mathrm{X}$ & $\mathrm{X}$ & $\mathrm{X}$ \\
\hline Socio-ecological measures & & & & $\mathrm{X}$ \\
\hline$C D 4 \& V L$ & & $\mathrm{X}$ & $\mathrm{X}$ & $\mathrm{X}$ \\
\hline
\end{tabular}

Fig. 1 WiseApp schematic diagram

and power calculation: a $75 \%$ retention rate by the end of the trial for both the control and intervention arms and that each person is on a once-daily regimen, a conservative assumption of high intraclass correlation coefficient (ICC) of 0.5 for same participant at different times, and the adherence rate is less than or equal to $80 \%$ at baseline. All calculations are based on a 2-sided test with alpha at 0.05 levels and power calculations being based on ART adherence.
Primary study data will be analyzed after completion of data collection, with study findings disseminated in peer-review public health journals.

\section{Data analysis for $R C T$}

Intention-to-treat principle will be applied for the primary outcome analysis. We purpose using a generalized linear mixed model (GLMM) to analyze primary outcome (adherence and secondary outcomes). For 


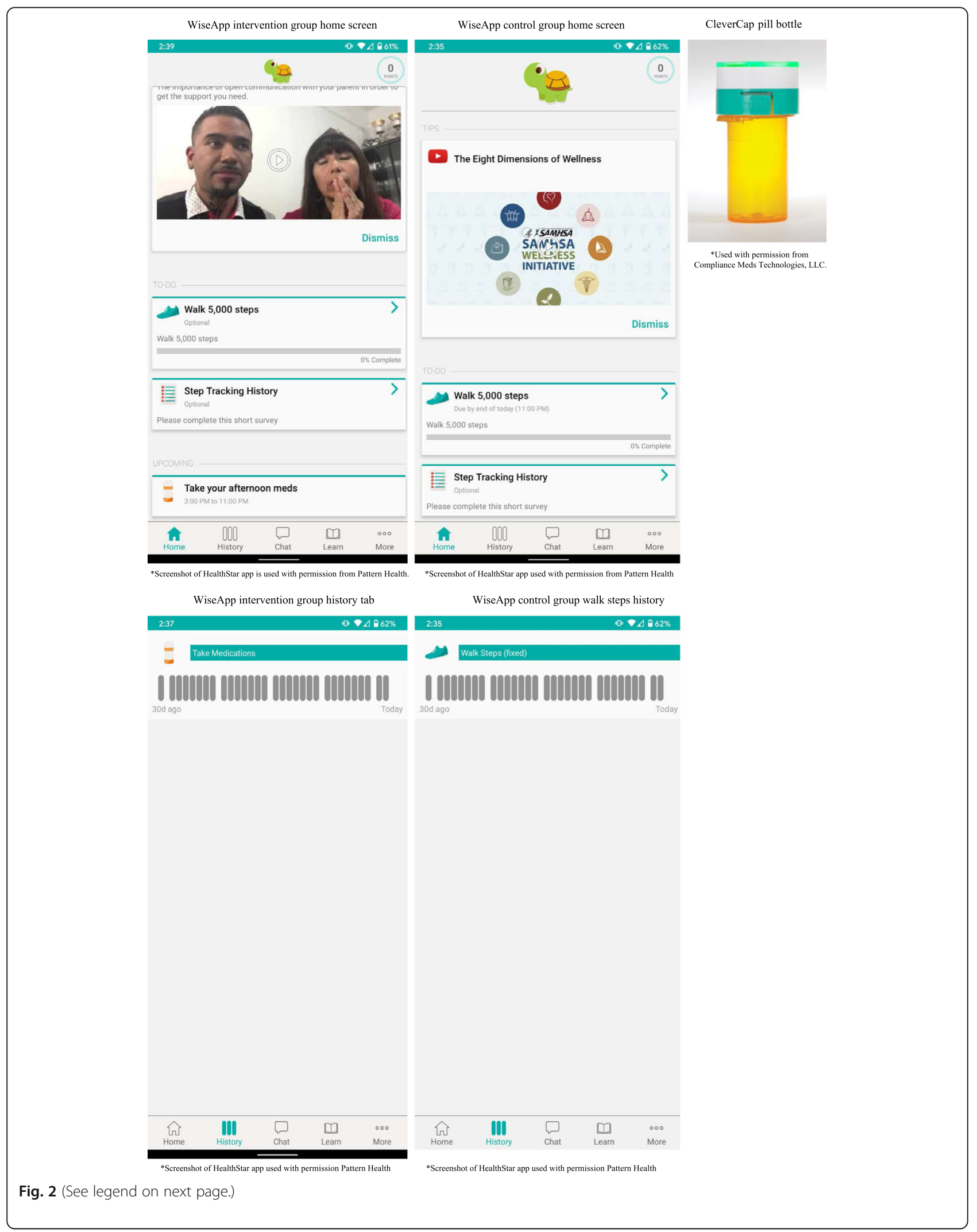


(See figure on previous page.)

Fig. 2 WiseApp study materials: WiseApp, CleverCap pill bottle, and fitness tracker. The intervention group receives videos and health surveys; all centered on medication adherence and managing living with HIV. A summary comparing the intervention vs. the control group is outlined below in Table 1. The intervention group's "To Do" list serves as daily reminders for medication adherence tracking, walk steps, and completing their weekly videos and surveys. As mentioned, the CleverCap pill bottle and the fitness tracker are both linked to the WiseApp. The history tab on the WiseApp shows the intervention group's medication adherence with green bars showing that they completed taking their medication for the day and red bars showing when they missed a day (Fig. 2). They receive daily app notification reminders for taking their medication

adherence outcome, a GLMM with logit link function will be used. In this model, independent variables are time (t) at three time points (baseline, 3 month, and 6 month), intervention arm, and interaction term between time and intervention. For the secondary outcomes in the analysis of the surveys related to the SDT framework, a similar GLMM with identity link function for continuous outcomes. In the analysis of app use, the unit of analysis will be at daily level for each participant, and system use will be analyzed using a GLMM with log link function (Poisson model).

For CD4 and viral load, we propose using GLMM with log link function because these variables are treated as count outcomes. Viral load will also be treated as a binary outcome (detectable vs. undetectable) and analyses of viral suppression will use the GLMM with logit link. In a secondary "as treated" analyses, missing adherence and viral load data will be ignored. Finally, we will assess the relationship between adherence (independent variable) and virologic suppression (dependent variable) using GLMM logistic regression and including missing viral load as detectable. This model will include personal level factors as covariates so we can test for potential confounding by covariates (i.e., age, gender, and health literacy) and inspect for changes in the point estimate of the relationship between study group and adherence.

Table 2 Study measures

\begin{tabular}{|c|c|c|}
\hline & Tool & $\begin{array}{l}\text { Measurement time } \\
\text { points }\end{array}$ \\
\hline $\begin{array}{l}\text { Descriptive Data: Socio-demographic } \\
\text { characteristics }\end{array}$ & Gender, age, education, income, employment, health insurance, housing & Baseline \\
\hline Descriptive Data: Behavioral Data & $\begin{array}{l}\text { Condom Use, Tobacco Use, Alcohol Use (AUDIT-C) [21], Substance Use } \\
{[22]}\end{array}$ & Baseline, 3 and 6 months \\
\hline Descriptive Data: Health Literacy & $\begin{array}{l}\text { Newest Vital Sign [23], Short Test of Functional Health Literacy in Adults } \\
\text { (S-TOFHLA) [24] }\end{array}$ & Baseline \\
\hline Descriptive Data: Health Status & $\begin{array}{l}\text { RAND-36 [25]; Symptom Distress Module [26]; PROMIS-29 [27]; Self- } \\
\text { Management Scale [28]; Baecke Questionnaire for the Elderly [29] }\end{array}$ & Baseline, 3 and 6 months \\
\hline Descriptive Data: Social Desirability & Social desirability scale [30] & Baseline \\
\hline Secondary Outcome: Physical Health Measures & $\begin{array}{l}\text { Height, Weight, and NHANES waist and hip measurement } \\
\text { protocol }[31,32] \text {. }\end{array}$ & Baseline, 3 and 6 months \\
\hline Descriptive Data: Housing Status & Housing Status Assessment Tool [33] & Baseline, 3 and 6 months \\
\hline \multirow[t]{3}{*}{ Primary Outcome: ART Adherence } & \multirow{3}{*}{$\begin{array}{l}\text { Medication Adherence measured through the CleverCap }{ }^{\mathrm{TM}} \text { Lite Dispenser } \\
\text { Pillbox }\end{array}$} & Daily \\
\hline & & Baseline, 3 and 6 months \\
\hline & & Baseline, 3 and 6 months \\
\hline \multirow[t]{2}{*}{ Secondary Outcomes: ART adherence } & CASE Adherence Index [34] & \\
\hline & CD4 and viral load & \\
\hline \multirow[t]{3}{*}{ Secondary Outcome: Health Care Access } & Number of Primary Care Visits & Baseline, 3 and 6 months \\
\hline & Engagement with Health care Provider Scale [35] & Baseline, 3 and 6 months \\
\hline & Caregiver Survey & 6 months \\
\hline Secondary Outcome: System Use & Automated Log Files & Ongoing \\
\hline \multirow[t]{4}{*}{ Secondary Outcome: Technology Acceptance } & Health-ITUES [36]; PSSUQ [37]; & Baseline, 3 and 6 months \\
\hline & Perceived Ease of Use and Potential Usefulness Questionnaire [38]; & \\
\hline & eHEALS: the Health Literacy Scale [39] & 3 months \\
\hline & Trust in Health Information Sources [40] & 6 months \\
\hline $\begin{array}{l}\text { Secondary Outcome: Social-Ecological } \\
\text { Measures }\end{array}$ & $\begin{array}{l}\text { Neighborhood Environment Survey [41], Social Capital Scale [42], \& } \\
\text { Self- Efficacy Scale [43] }\end{array}$ & 6-months \\
\hline
\end{tabular}


Primary study data will be analyzed as soon as possible after the end of data collection, with study findings disseminated in peer-review public health journals.

\section{Discussion}

This protocol describes a randomized controlled efficacy trial using a mHealth intervention to help improve ART adherence and overall quality of life of PLWH living in NYC. Previously, mHealth tools to help with HIV management, specifically adherence, have been primarily studied outside the United States in low and middleincome countries $[45,46]$. A strength of our study is that, to our knowledge, this is one of the first randomized controlled trials assessing a mHealth application intervention for medication adherence and HIV management in the United States. The app was developed through user-centered design, having end-users intricately involved in the design iteration process with the only feature of the app being manipulated being the adherence features, controlling for participants receiving a health app [14]. Another strength of the WiseApp trial is the use of real-time medication monitoring with the Clever-Cap pill dispenser. This real-time monitoring can allow for easier detection of lapses in adherence and could potentially help providers initiate interventions to help resume treatment before drug resistance begins, something not previously done [47].

However, as a randomized controlled trial, there are limitations, such as detecting if differences in usage of the intervention affected outcomes. Additionally, part of this trial is occurring during the global COVID-19 pandemic. This pandemic could affect people's usage of the app intervention due to extenuating outside stressors drawing their attention away. Some participants have also expressed difficulty in renewing medication during the pandemic.

Despite the limitations that come with an RCT, the WiseApp trial has the potential to fill a gap in the mHealth literature and provide new knowledge regarding the effectiveness of mobile app interventions for PLWH in the United States. A systematic literature review of impact studies of mHealth for HIV treatment has already found that mHealth can be effective in improving ART adherence in low and middle-income countries [46]. In another review of 721 articles, there were only six studies that reported mHealth interventions assisting with antiretroviral (ART) medication adherence among HIV-positive men who have sex with men [48]. Of the six studies, only two used mobile applications for ART and are only in the pilot phase and the beginning phases of a RCT [48]. If successful this study will not only expand the literature but also bring new advances for reaching underserved populations in the United States, improving both their adherence to HIV treatment regimens and advancement in the HIV treatment cascade.

\section{Supplementary information}

Supplementary information accompanies this paper at https://doi.org/10. 1186/s12889-020-09688-0.

Additional file 1. Wise App Trial Baseline.

\section{Abbreviations}

PLWH: Persons living with HIV; HIV: Human immunodeficiency virus; ART: Antiretroviral therapy; RCT: Randomized control trial; GLMM: Generalized linear mixed model; CUIMC: Columbia University Irving Medical Center; VL: Viral load

\section{Acknowledgements}

Not applicable.

\section{Authors' contributions}

RS contributed to the design of all aspects of the study. RS, NR, and DCM contributed to the proposed study design. HJ designed the proposed data analysis. GF and RS drafted the manuscript. All authors have read and approved the final manuscript.

\section{Authors' information}

Not applicable.

\section{Funding}

This study was funded by the Agency for Healthcare Research and Quality, Grant/Award Number: R01HS025071 and the National Institute of Nursing Research, Grant/ Award Number: K24NR018621. The content is solely the responsibility of the authors and does not necessarily represent the official views of AHRQ and the NIH. The funding source has no role in the original design of this study, analysis and interpretation of data, or decision to submit results.

\section{Availability of data and materials \\ Not applicable.}

\section{Ethics approval and consent to participate}

This protocol has been approved by the Institutional Review Board of Columbia University (IRB-AAAQ9957) with written informed consent. Study participants complete a written informed consent prior to participation in research activities. Current protocol version: September 4, 2019. Any modifications to the protocol are submitted to and approved by the IRB prior to implementation. Spontaneously reported adverse events and unintended effects of the trial are tracked by the study Principal Investigators and reported to the IRB. This study is monitored by a Data Safety and Monitoring Board (DSMB), the composition of which may be obtained from the study principal investigator. No criteria for discontinuing or modifying the intervention or trial stopping rules were defined for this study.

\section{Consent for publication}

Not applicable.

\section{Competing interests}

The authors declare that they have no competing interests.

\section{Author details}

${ }^{1}$ College of Public Health, University of South Florida, 13201 Bruce B. Downs Blvd, Tampa, FL 33612, USA. '2Division of Scholarship and Research, Columbia University School of Nursing, 560 West 168th Street, New York, NY 10032, USA. J Johns Hopkins University School of Nursing, 525 N Wolfe Street, Baltimore, MD 21205, USA. ${ }^{4}$ Center for Behavioral Intervention Technologies, Northwestern University, 750 N Lake Shore, Chicago, IL 60611, USA.

Received: 1 September 2020 Accepted: 13 October 2020

Published online: 25 November 2020

\section{References \\ 1. HIV Basic Statistics, https://www.cdc.gov/hiv/basics/statistics.html (2020, Accessed May 14 2020).}


2. Deeks SG, Lewin SR, Havlir DV. The end of AIDS: HIV infection as a chronic disease. Lancet. 2013:382:1525-33 DOI: https://doi.org/10.1016/S01406736(13)61809-7.

3. Gardner EM, McLees MP, Steiner JF, et al. The Spectrum of engagement in HIV care and its relevance to test-and-treat strategies for prevention of HIV infection. Clin Infect Dis. 2011;52:793-800. https://doi.org/10. 1093/cid/ciq243.

4. Murri R, Ammassari A, Gallicano K, et al. Patient-reported nonadherence to HAART is related to protease inhibitor levels. J Acquired Immune Deficiency Syndromes. 2000;24:123-8 Article.

5. Mugavero MJ, Lin HY, Allison JJ, et al. Failure to establish HIV care: characterizing the "no show" phenomenon. Clin Infect Dis 2007; 45: 127130. 2007/06/08. DOI: https://doi.org/10.1086/518587.

6. Mills EJ, Nachega JB, Bangsberg DR, et al. Adherence to HAART: a systematic review of developed and developing nation patient-reported barriers and facilitators. PLoS Med 2006; 3: e438. 2006/11/24. DOl: https://doi.org/10. 1371/journal.pmed.0030438.

7. NYS HIV Surveillance System. HIV care continuum - people living with HIV, New York state (2018). 2019.

8. Golin CE, Liu H, Hays RD, et al. A prospective study of predictors of adherence to combination antiretroviral medication. J General Internal Med 2002; 17: 756-765. Article. DOI: https://doi.org/10.1046/j.1525-1497. 2002.11214.x.

9. Bangsberg DR, Acosta EP, Gupta $R$, et al. Adherence-resistance relationships for protease and non-nucleoside reverse transcriptase inhibitors explained by virological fitness. AIDS 2006; 20: 223-231. 2006/03/03. DOI: https://doi. org/10.1097/01.aids.0000199825.34241.49.

10. Wainberg MA and Friedland G. Public health implications of antiretroviral therapy and HIV drug resistance. JAMA 1998; 279: 1977-1983. 1998/06/27. DOI: https://doi.org/10.1001/jama.279.24.1977.

11. Kumar S, Nilsen WJ, Abernethy A, et al. Mobile health technology evaluation: the mHealth evidence workshop. Am J Prev Med 2013; 45: 228236. 2013/07/23. DOI: https://doi.org/10.1016/j.amepre.2013.03.017.

12. Estrin D and Sim I. Health care delivery. Open mHealth architecture: an engine for health care innovation. Science 2010; 330: 759-760. 2010/11/06. DOl: https://doi.org/10.1126/science.1196187.

13. Schnall R, Rojas M, Travers J, et al. Use of Design Science for Informing the Development of a Mobile App for Persons Living with HIV. AMIA Annual Symposium proceedings AMIA Symposium 2014; 2014: 1037-1045. 2014/01/01.

14. Beauchemin M, Gradilla M, Baik D, et al. A Multi-step Usability Evaluation of a Self-Management App to Support Medication Adherence in Persons Living with HIV. Int J Med Informatics 2019; 122: 37-44. DOI: https://doi.org/ 10.1016/j.jmedinf.2018.11.012.

15. Folstein MF, Folstein SE and McHugh PR. "Mini-mental state": A practical method for grading the cognitive state of patients for the clinician. J Psychiatric Res 1975; 12: 189-198. DOl: https://doi.org/10.1016/00223956(75)90026-6.

16. Altman DG and Bland JM. Statistics notes. Treatment allocation in controlled trials: why randomise? BMJ 1999; 318: 1209. 1999/04/30. DOI: https://doi. org/10.1136/bmj.318.7192.1209

17. Haberer JE, Kahane J, Kigozi I, et al. Real-time adherence monitoring for HIV antiretroviral therapy. AIDS Behav. 2010;14:1340-6. https://doi.org/10.1007/ s10461-010-9799-4.

18. Fogg BJ. Persuasive technology: using computers to change what we think and do. Ubiquity 2002; 2002: Article 5. DOI: https://doi.org/10. 1145/764008.763957.

19. Tudor-Locke C, Craig CL, Thyfault JP, et al. A step-defined sedentary lifestyle index: <5000 steps/day. Appl Physiol Nutr Metab. 2012;38:100-14. https:// doi.org/10.1139/apnm-2012-0235.

20. Tudor-Locke C, Bassett DR. How many steps/day are enough? Sports Med. 2004;34:1-8. https://doi.org/10.2165/00007256-200434010-00001.

21. Bush K, Kivlahan DR, McDonell MB, et al. The AUDIT alcohol consumption questions (AUDIT-C): an effective brief screening test for problem drinking. Arch Intern Med. 1998;158:1789-95.

22. American Psychiatric Association Adapted NIDA Modified ASSIST Tools, https://www.drugabuse.gov/nidamed-medical-health-professionals/ screening-tools-resources/american-psychiatric-association-adapted-nidamodified-assist-tools (2015)

23. Weiss $B D$, Mays $M Z$, Martz $W$, et al. Quick assessment of literacy in primary care: the newest vital sign. Ann Fam Med 2005; 3: 514-522. 2005/12/13. DOI: https://doi.org/10.1370/afm.405.
24. Parker RM, Baker DW, Williams MV, et al. The test of functional health literacy in adults: a new instrument for measuring patients' literacy skills. J Gen Intern Med 1995; 10: 537-541. 1995/10/01. DOI: https://doi.org/10.1007/ bf02640361.

25. Hays RD, Sherbourne CD and Mazel RM. The RAND 36-Item Health Survey 1. 0. Health Econ 1993; 2: 217-227. 1993/10/01. DOl: https://doi.org/10.1002/ hec.4730020305.

26. Marc LG, Wang MM and Testa MA. Psychometric evaluation of the HIV symptom distress scale. AIDS Care 2012; 24: 1432-1441. 2012/03/14. DOI: https://doi.org/10.1080/09540121.2012.656567.

27. Viney R, Norman R, Brazier J, et al. An Australian discrete choice experiment to value eq-5d health states. Health Econ 2014; 23: 729-742. 2013/06/15. DOI: https://doi.org/10.1002/hec.2953.

28. Webel AR, Asher A, Cuca Y, et al. Measuring HIV self-management in women living with HIV/AIDS: a psychometric evaluation study of the HIV Self-management Scale. J Acquir Immune Defic Syndr 2012; 60: e72-81. 2012/05/10. DOl: https://doi.org/10.1097/QAl.0b013e318256623d.

29. Hertogh EM, Monninkhof EM, Schouten EG, et al. Validity of the modified Baecke questionnaire: comparison with energy expenditure according to the doubly labeled water method. Int J Behav Nutr Phys Act 2008; 5: 30. 2008/05/29. DOl: https://doi.org/10.1186/1479-5868-5-30.

30. Reynolds WM. Development of reliable and valid short forms of the MarloweCrowne social desirability scale. J Clin Psychol. 1982;38:119-25. https://doi.org/ 10.1002/1097-4679(198201)38:1<119::AID-JCLP2270380118>3.0.CO;2-I.

31. National Health and Nutrition Examination Survey III (NHANES III). Body Measurements (Anthropometry).

32. Centers for Disease Control and Prevention NCfHS. National Health and Nutrition Examination Study (NHANES) Anthropometry Procedures Manual. Hyattsville, MD: U.S. Department of Health and Human Services: Department of Health and Human Services, Centers for Disease Control and Prevention, 2007-2008.

33. Albanese T, Wood M, Spellmna B. Housing status assessment guide for state TANF and Medicaid programs. Bethesda, MD: U.S. Department of Health and Human Services; 2009.

34. Mannheimer SB, Mukherjee R, Hirschhorn LR, et al. The CASE adherence index: A novel method for measuring adherence to antiretroviral therapy. AIDS Care 2006; 18: 853-861. 2006/09/15. DOl: https://doi.org/10.1080/ 09540120500465160

35. Bakken S, Holzemer WL, Brown MA, et al. Relationships between perception of engagement with health care provider and demographic characteristics, health status, and adherence to therapeutic regimen in persons with HIV/ AIDS. AIDS Patient Care STDS 2000; 14: 189-197. 2000/05/12. DOI: https:// doi.org/10.1089/108729100317795.

36. Schnall R, Cho H, Liu J. Health Information Technology Usability Evaluation Scale (Health-ITUES) for Usability Assessment of Mobile Health Technology: Validation Study. JMIR Mhealth Uhealth. 2018;6:e4. 2018/01/07. https://doi. org/10.2196/mhealth.8851.

37. Lewis JR. Psychometric evaluation of the PSSUQ using data from five years of usability studies. Int J Human-Computer Interaction. 2002;14:463-88. https://doi.org/10.1080/10447318.2002.9669130.

38. Davis FD. Perceived usefulness, perceived ease of use, and user acceptance of information technology. MIS Q. 1989;13:319-40. https:// doi.org/10.2307/249008.

39. Norman CD and Skinner HA. eHEALS: The eHealth Literacy Scale. J Med Internet Res 2006; 8: e27. 2007/01/11. DOl: https:/doi.org/10.2196/jmir.8.4.e27.

40. Chen X, Hay JL, Waters EA, et al. Health literacy and use and Trust in Health Information. J Health Commun. 2018;23:724-34. https://doi.org/10.1080/ 10810730.2018.1511658.

41. Rosenberg D, Ding D, Sallis JF, et al. Neighborhood Environment Walkability Scale for Youth (NEWS-Y): Reliability and relationship with physical activity. Preventive Medicine. 2009;49:213-8. https://doi.org/10.1016/j.ypmed.2009.07.011.

42. O'Brien MS, Burdsal CA, Molgaard CA. Further development of an Australianbased measure of social capital in a US sample. Social Sci Med. 2004;59: 1207-17. DOI. https://doi.org/10.1016/j.socscimed.2004.01.007.

43. Resnick B, Jenkins LS. Testing the reliability and validity of the self-efficacy for exercise scale. Nurs Res. 2000;49.

44. Research NloN. The NINR strategic plan: advancing science, improving lives. 2016.

45. Sidney $\mathrm{K}$, Antony J, Rodrigues $\mathrm{R}$, et al. Supporting patient adherence to antiretrovirals using mobile phone reminders: patient responses from South India. AIDS Care. 2012;24:612-7. https://doi.org/10.1080/09540121. 2011.630357 
46. Catalani C, Philbrick W, Fraser $H$, et al. mHealth for HIV Treatment \& Prevention: a systematic review of the literature. Open AIDS J. 2013;7:17-41. https://doi.org/10.2174/1874613620130812003.

47. Bangsberg DR, Deeks SG. Spending more to save more: interventions to promote adherence. Ann Intern Med. 2010;152:54-13. https://doi.org/10. 7326/0003-4819-152-1-201001050-00012.

48. Muessig KE, LeGrand S, Horvath KJ, et al. Recent mobile health interventions to support medication adherence among HIV-positive MSM. Curr Opin HIV AIDS. 2017;12:432-41. https://doi.org/10.1097/COH.0000000000000401.

\section{Publisher's Note}

Springer Nature remains neutral with regard to jurisdictional claims in published maps and institutional affiliations.

Ready to submit your research? Choose BMC and benefit from:

- fast, convenient online submission

- thorough peer review by experienced researchers in your field

- rapid publication on acceptance

- support for research data, including large and complex data types

- gold Open Access which fosters wider collaboration and increased citations

- maximum visibility for your research: over $100 \mathrm{M}$ website views per year

At BMC, research is always in progress.

Learn more biomedcentral.com/submissions 